\title{
Application of CBNAAT (Xpert MTB/RIF assay) in new smear negative pulmonary tuberculosis patients
}

\author{
Vishal Chopra, Baljeet Singh Virk, Siddharth Chopra, Monika Bansal, Jain Chungath \\ Department of Pulmonary Medicine, Government Medical College, Patiala, India
}

\begin{abstract}
Presumptive pulmonary tuberculosis (PTB) patients whose sputum are detected to be smear negative for acid fast bacilli (AFB) present a significant challenge for a treating physician. Initiating these patients on anti tuberculous treatment (ATT) on empirical basis is not a good strategy as many were found to be sputum culture for tuberculosis negative on further evaluation according to many previous studies. In India due to resource limited settings and lack of knowledge about newest diagnostic modalities patients are often initiated only on the basis of characteristic clinical symptoms and chest radiographic abnormalities. This study was conducted to identify the advantage of application of sputum cartridge based nucleic acid amplification test (CBNAAT) in sputum AFB smear negative presumptive pulmonary TB patients. Our study concluded
\end{abstract}

Correspondence: Vishal Chopra, 27 Bank Colony, 147001Patiala, Punjab, India.

Mobile: +91.9814146788

E-mail: drvishalchopra@hotmail.com

Conflict of interest: The authors declare no conflict of interest.

Contributions: All the Authors contributed equally. All the authors have read and approved the final version of the manuscript and agreed to be accountable for all aspects of the work.

Availability of data and materials: All data generated or analyzed during this study are included in this published article.

Ethics approval: The study was done after ethical approval the Institutional Review Board.

Informed consent: Informed consent was taken from all the patients.

Consent for publication: Not applicable.

Key words: CBNAAT; smear negative; Xpert MTB.

Received for publication: 10 August 2019.

Accepted for publication: 2 January 2020.

COpyright: the Author(s), 2020

Licensee PAGEPress, Italy

Monaldi Archives for Chest Disease 2020; 90:1146

doi: 10.4081/monaldi.2020.1146

This article is distributed under the terms of the Creative Commons Attribution Noncommercial License (by-nc 4.0) which permits any noncommercial use, distribution, and reproduction in any medium, provided the original author(s) and source are credited. that clinical symptoms and radiological characteristics cannot differentiate TB patients from non-TB patients. Treating patients only on empirical basis would have resulted in unnecessary treatment of 41 patients.

\section{Introduction}

Presumptive pulmonary tuberculosis (PTB) is a major health problem in India with significant morbidity and mortality. As per the World Health Organisation Global TB Report 2019, the annual incidence of tuberculosis in India was 2.7 million with mortality of 449,000 cases [1]. The first step in diagnosis of PTB is made by sputum microscopy along with chest radiography under Revised National Tuberculosis Control Programme (RNTCP) in patients with presumptive Koch. Sputum smear examination is simple, economical, requires only minimum training of laboratory technicians and is freely available at all health facilities and the results are available on the same day. But its main drawback is that there must be at least 5000-10,000 acid fast bacilli (AFB) per mm of specimen for detection of bacteria [2]. It has low sensitivity (22$82 \%$ ), high specificity (99-99.9\%), false negative reporting of 17.6 to $78 \%$ and is unable to detect drug resistance [3]. A low bacillary load leads to a smear negative PTB, an area of diagnostic dilemma which results in a delay in diagnosis and continued transmission of TB. According to WHO 2009, AFB are directly identifiable using smear microscopy in only less than half of the patients with PTB [4]. For smear negative cases WHO had recommended sputum mycobacterial culture plus chest radiography [5]. It has been found that $27 \%$ of patients who were sputum smear negative for AFB were detected culture positive for Mycobacterium tuberculosis (MTB). In another significant study it was found that up to $48 \%$ cases were missed by only sputum smear examination and were later detected by culture. Also out of those patients who were started empirically on ATT only $38 \%$ had culture positive for MTB [6].17\% out of the smear negative, culture positive patients have been proven to transmit tuberculosis [7]. In immune-compromised patients especially HIV infected patients with pulmonary TB, the rate of negative result on sputum smear examination for acid-fast bacilli is higher and can range from 24-61\% [8]. All these factors make diagnosis in patients with a presumptive TB who turn out to be sputum smear negative for AFB a clinical challenge.

Conventional mycobacterial culture (Lowenstein-Jensen medium or Middle-brook medium) is considered as the gold standard for diagnosis of TB but results are available after 4 weeks with an additional time required for drug susceptibility testing [9]. In liquid culture i.e. Mycobacterial Growth Indicator Tube (MGIT), which is a faster culture method, the results are available in 2 weeks but needs a well-trained microbiologist, costly infrastructure and 
strict biosafety requirements which are not feasible at peripheral health care facilities in India .

A rapid, accurate and reliable diagnostic test is needed in these patients so that early anti tuberculous treatment (ATT) can be started for preventing the transmission of TB and clinical severity of the disease. Furthermore, unnecessary treatment can be avoided in patients who do not have TB with avoidance of additional expenditure on medication. The advent of gene Xpert has resulted in a paradigm shift in the diagnosis of TB [10). This study was conducted in an intermediate reference lab in a tertiary care hospital in north India to evaluate the role of CBNAAT in diagnosing TB in sputum smear negative patients of presumptive pulmonary $\mathrm{TB}$.

\section{Patients and Methods}

The present study was conducted with the Institutional Review Board and Institutional Ethics Committee approval. It was conducted in an intermediate reference lab in a tertiary hospital in North India from January 2018 to January 2019. It was a prospective observational study which included 100 consecutive patients presenting with clinical symptoms and radiographic abnormalities consistent with pulmonary tuberculosis, but having sputum smear negative for AFB and no previous anti-tubercular treatment records.

\section{Inclusion criteria}

Patients with symptoms consistent with TB i.e. persistent cough for $\geq 2$ weeks, fever, night sweats, loss of appetite, weight loss of $\geq 1.5 \mathrm{~kg}$ in a month, malaise, shortness of breath, chest pain, and having chest radiograph abnormalities consistent with TB i.e. infiltrates, cavity, opacity, lung abscess, miliary shadows, and sputum smears were negative for AFB.

\section{Exclusion criteria}

Patients who had sputum smear positive for AFB and had been treated for tuberculosis in the past were excluded.

\section{Study sequence}

Informed consent was obtained from the patients. The patients having two sputum smears detected negative for AFB, were asked to collect two sputum samples in falcon tubes for CBNAAT (Xpert MTB/RIF) testing at Intermediate reference laboratory (IRL) in this hospital. Those patients in whom MTB was not detected on CBNAAT, the sputum were subjected further to liquid culture (MGIT).

The results of observations of individual patients were pooled and analysed. Statistical analysis was performed using SPSS software ver. 20.0 Chicago, IL, USA. The $p<0.05$ was considered statistically significant and $\mathrm{p}<0.01$ was considered as highly significant statistically. For categorical variables chi-square test was used for analysis.

\section{Results}

Out of 100 patients included in the study 43 were males and 57 females. In the CBNAAT positive group the number of males and females were the same $(\mathrm{n}=29)$ and in the negative group, females outnumbered the males, $\mathrm{n}=28$ and 14 (66.6 and $33.3 \%$ ), respectively.

The mean age of patients in both the group was $42 \pm 19.41$ years. In the CBNAAT MTB detected group maximum number of patients were from the age group $21-30$ years, $n=13(22.40 \%)$ whereas in the not detected group they were from 51-60 years' age group, $n=11$ $(26.19 \%)$.

The most common clinical feature was cough among both CBNAAT detected and not detected group (100\% in both groups). But results were found to be not statistically significant. The only statistically significant clinical features were mucoid sputum production $(\mathrm{n}=35,76.08 \%$ vs $\mathrm{n}=28,73.68 \%, \mathrm{p}=0.01)$ and evening rise of temperature $(\mathrm{n}=33,56.8$ vs $\mathrm{n}=14,33.3 \%, \mathrm{p}=0.02)$. The other features where found to have the following frequencies and percentages in CBNAAT positive group $v s$ negative group as described below: fever $(\mathrm{n}=42,72.4 \%$ vs $\mathrm{n}=32,76.1 \%)$, night sweats $(\mathrm{n}=23$, $39.6 \%$ vs $\mathrm{n}=32,76.19 \%)$, loss of weight $(\mathrm{n}=40,68.96 \%$ vs $\mathrm{n}=20$, $47.61 \%)$, loss of appetite $(\mathrm{n}=43,74.1 \%$ vs $\mathrm{n}=25,59.5 \%)$, malaise $(\mathrm{n}=40,68.96 \%$ vs $\mathrm{n}=27,64.2 \%)$, shortness of breath $(\mathrm{n}=38,65.5 \%$ vs $\mathrm{n}=27,64.2 \%)$ and chest pain $(\mathrm{n}=18,31 \%$ vs $\mathrm{n}=19,45.2 \%)$. These values were also found to be statistically insignificant (Figure 1).

The radiographic findings were also similar in both the groups and all were statistically non-significant. The most common radiographic finding in the CBNAAT detected group was opacity $(\mathrm{n}=29,50 \%)$ followed by infiltrates $(\mathrm{n}=18,31 \%)$, nodules $(\mathrm{n}=7$, $12.06 \%)$, cavity $(n=5,8.6 \%)$ and miliary nodules $(n=4,6.8 \%)$. In CBNAAT negative group also similar trend was seen, opacity was the most common finding $(\mathrm{n}=19,45.2 \%)$ followed by infiltrates $(n=14,33.3 \%)$, cavity $(n=7,16.6 \%)$, and nodules $(n=1,2.3 \%)$. It was observed that cavitary lesion was seen more in CBNAAT negative group (8.6\% vs 16.6\%) (Figure 2).

We could not differentiate TB patients from those without TB on basis of clinical features and radiographic findings. There were important differences with respect to distribution of radiographic lesion in the different pulmonary zones between both the groups. The upper zones and mid-zones were predominantly involved in the MTB detected group and were statistically significant $(p<0.001)$. Lower zones were less commonly involved in MTB detected $(p<.01)$. There were statistically significant number of patients with all zones involved in MTB detected group compared to the MTB not detected group (Table 1). Bilateral involvement was more common in MTB detected group and was statistically significant $(\mathrm{p}<0.03)$.

Out of the cohort of 100 sputum smear negative patients, MTB was detected in 58 patients by CBNAAT and all were found to be rifampicin sensitive. Sputum of rest 42 patients was put on liquid culture out of which just one showed growth of mycobacterium tuberculosis. The growth was again subjected to CBNAAT, and it showed rifampicin sensitivity (Figure 3).

\section{Discussion}

In PTB patients whose results are smear negative for AFB despite significant chest radiography findings, there is a significant challenge for the treating physician for initiating treatment. Starting these patients on ATT empirically is not a good strategy as $60 \%$ of 
patients were found to be culture negative on further investigation. In India patients are often initiated treatment on the basis of symptoms and radiographic abnormalities. This study showed that clinical features and radiological characteristics cannot differentiate TB from Non-TB patients. Treating all the patients in our study would have resulted in 41 patients with unnecessary AT medication. Recently, a comparative study of Xpert MTB/RIF with ZiehlNeelsen staining was performed which demonstrated a higher sensitivity of Xpert MTB/RIF as compared to Ziehl-Neelsen staining in respiratory samples [11].

The result of this study also demonstrates that the prevalence of tuberculosis in sputum smear negative patients is considerably high $(58 \%)$ and unless subjected to sputum culture these cases would have been undetected. Many studies have shown a very high specificity of sputum CBNAAT $[12,13]$. Xpert Ultra is a newer technology in which very low bacillary loads can be detected which are labelled as traced but Rifampicin sensitivity cannot be measured. The sensitivity is more than Xpert by $5 \%$ but specificity is less by $3 \%$ and this test has been recommended by WHO to replace CBNAAT [14]. Latest rapid molecular detection techniques include melt pro TB assay and Xpert XDR assay, these are still under development and has not been endorsed by WHO yet [15].
It has also been shown in a recent meta-analysis has a pooled sensitivity of $89 \%$ and a specificity of $99 \%$ in diagnosis of pulmonary TB [13]. We did not perform any follow-up cultures following a positive CBNAAT result to confirm the diagnosis and ascertain the diagnostic accuracy like many previous studies because there are small chances of receiving false positive results [16] and also considering the high patient load and economic costs. In the absence of CBNAAT repeated sputum examinations will delay the diagnosis of TB and delay the treatment due to time lag for sputum culture results. CBNAAT results are available within few hours and helps in initiating ATT on the same day, thus chances of patients being lost to follow up is decreased. Only those patients in whom MTB is not detected on CBNAAT can be followed with culture results. This will in prevent overuse of sputum culture as a diagnostic modality. This strategy also decrease disease transmission.

The impact of delayed detection of TB has been rightly pointed out as there is increased morbidity and permanent damage to lungs in patients, increased transmission within the community and the increased economic impact due to loss of earning and increased health care visits. Hence early diagnosis of TB has an overall economic benefit on individual, family and society. Another advantage of CBNAAT is also detection of Rifampicin resistance

Table 1. Zones involved on chest radiography.

\begin{tabular}{|c|c|c|c|c|c|}
\hline & CBNAAT-MTB-detected $(\mathrm{n}=58)$ & CBNAAT-MTB-not detected $(\mathrm{n}=42)$ & $\mathrm{X}^{2}$ & p-value & \\
\hline Upper zone & $17(29.31 \%)$ & $7(16.66 \%)$ & 12.25 & 0.001 & $\mathrm{HS}$ \\
\hline Midzone & $14(24.13 \%)$ & $13(30.95 \%)$ & 0.02 & 0.483 & NS \\
\hline Lower zone & $5(8.60 \%)$ & $13(30.95 \%)$ & 7.36 & 0.010 & S \\
\hline Upper + mid zones & $13(22.41 \%)$ & $4(9.52 \%)$ & 11.12 & 0.001 & HS \\
\hline Mid + lower zones & $3(5.17 \%)$ & $5(11.90 \%)$ & 0.16 & 0.513 & NS \\
\hline All zones & $15(25.86 \%)$ & $4(9.5 \%)$ & 4.70 & 0.010 & S \\
\hline Bilateral & $24(70.58 \%)$ & $10(29.41 \%)$ & 7.65 & 0.032 & S \\
\hline
\end{tabular}

HS, highly significant; NS, not significant; S, significant.

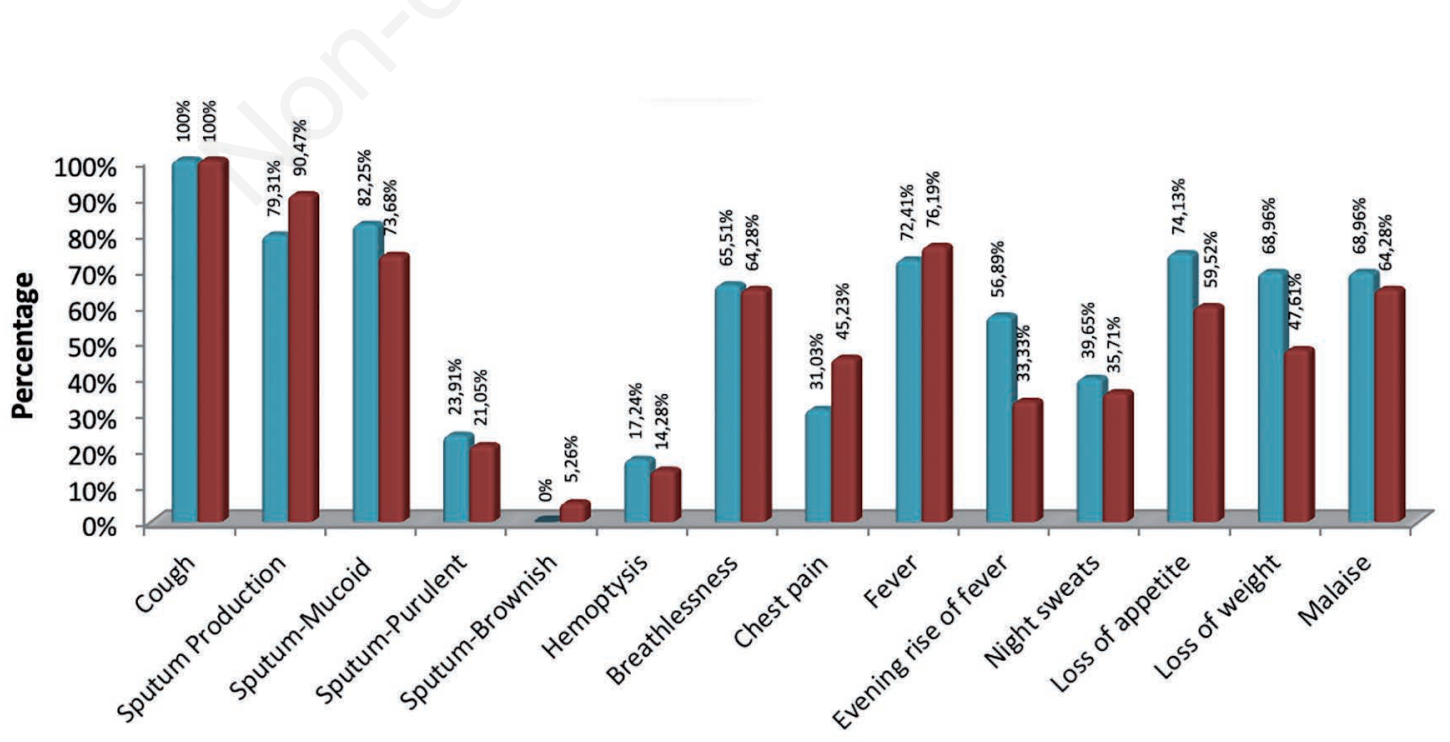

$\square$ CBNAAT MTB detected $\square$ CBNAAT MTB not detected

Figure 1. Clinical features. 
which is becoming a big problem in high prevalence countries. Though this test has great importance in India but it had been pointed out that providing this test to all smear negative TB suspects would consume the entire national health care budget [17]. It should be understood that CBNAAT does not have high sensitivity as culture and the clinician should not use this test to exclude the diagnosis of TB. Before using the test in regular basis the clinicians should be well informed about the limitations of this test [18].

In our study only 58 out of 100 patients were detected by CBNAAT which reflects low sensitivity which is in contrast to previous study which evaluated the sensitivity to be $100 \%$ [19]. The causes could be due to poor sputum sample quality and technical errors.

This study has certain limitations. It was performed at a regional TB centre with high prevalence of tuberculosis and the results cannot be generalized to all geographic locations. Second, we did not subject the sputum from CBNAAT positive cases for solid or liquid culture as the aim of this study was not to assess the sensitivity and specificity of CBNAAT, as it has been done earlier by various investigators.

\section{Conclusions}

Application of CBNAAT test in our tertiary referral centre had high specificity and supports a delayed ATT treatment in CBNAAT negative patients till culture results are available. Clinical judgment should be used when treating this subset of patients in case of rapidly worsening symptoms. We recommend CBNAAT in smear negative presumptive pulmonary TB patients so as to reach the diagnosis at the earliest thus curtailing the transmission of TB in community.

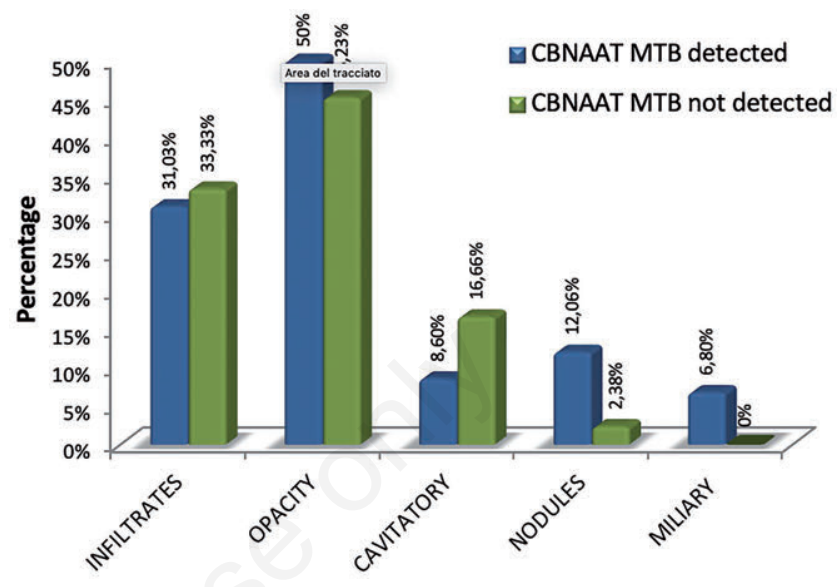

Figure 2. Radiological findings.

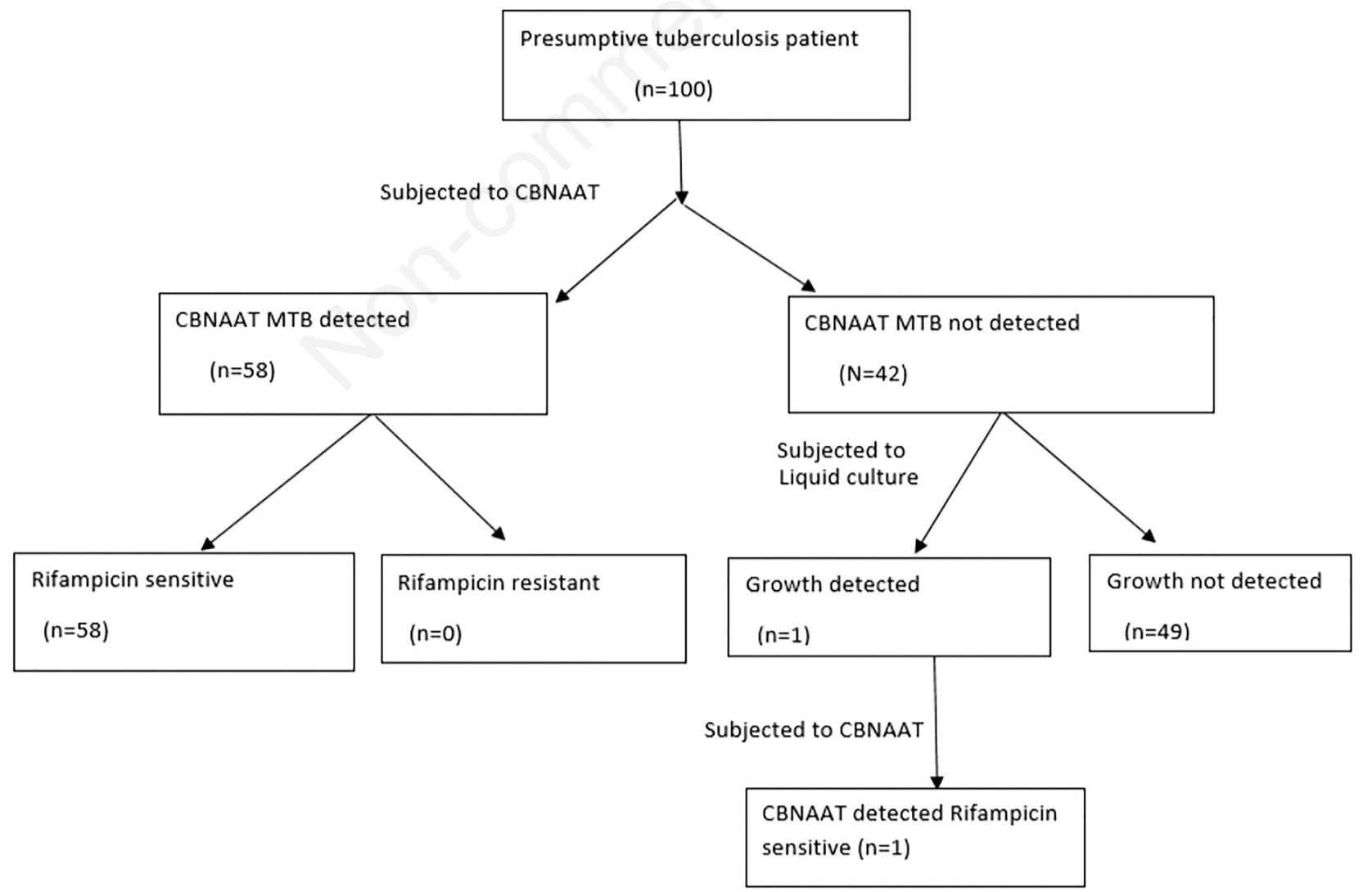

Figure 3. Flow diagram of the study sequence and patient cohort. 


\section{References}

1. WHO. Global tuberculosis report 2019. World Health Organization, Geneva; 2019.

2. Rouillon A, Perdrizet S, Parrot R. Transmission of tubercle bacilli: The effects of chemotherapy. Tubercle 1976;57:275-99.

3. Rickman TW, Moyer NP. Increased sensitivity of acid-fast smears. J Clin Microbiol 1980;11:618-20.

4. WHO. Global tuberculosis control: a short update to the 2009 report. World Health Organization, Geneva; 2009. Available at: https://apps.who.int/iris/handle/10665/44241

5. WHO. Improving the diagnosis and treatment of smearnegative pulmonary and extrapulmonary tuberculosis among adults and adolescents. Recommendations for HIV-prevalent and resource-constrained settings. World Health Organization, Geneva; 2007. Available at: https://www.who.int/hiv/pub/tb/ pulmonary/en/

6. Swai HF, Mugusi FM, Mbwambo JK. Sputum smear negative pulmonary tuberculosis: sensitivity and specificity of diagnostic algorithm. BMC Res Notes 2011;4:475.

7. Behr MA, Warren SA, Salamon H, et al. Transmission of Mycobacterium tuberculosis from patients smear-negative for acid-fast bacilli. Lancet 1999;353:444-9.

8. Harries AD, Maher D, Nunn P. An approach to the problems of diagnosing and treating adult smear-negative pulmonary tuberculosis in high-HIV-prevalence settings in sub-Saharan Africa. Bull World Health Organ 1998;76:651-62.

9. van Deun A. What is the role of mycobacterial culture in diagnosis and case definition? In: Frieden T, editor. Toman's tuberculosis case detection, treatment, and monitoring. 2nd ed. Geneva: World Health Organization; 2004; p. 35-43.

10. Varma-Basil M, Shah A. GeneXpert: A momentous innovation that needs a touch of prudence. Indian J Tuberc 2017;64:69-71.

11. Agrawal M, Bajaj A, Bhatia V, Dutt S. Comparative study of GeneXpert with ZN stain and culture in samples of suspected pulmonary tuberculosis. J Clin Diagn Res 2016;10:9-12.

12. Lawn SD, Mwaba P, Bates M, et al. Advances in tuberculosis diagnostics: the Xpert MTB/RIF assay and future prospects for a point-of-care test. Lancet Infect Dis 2013;13:349-61.

13. Steingart KR, Schiller I, Horne DJ, et al. Xpert ${ }^{\circledR}$ MTB/RIF assay for pulmonary tuberculosis and rifampicin resistance in adults. Cochrane Database Syst Rev 2014:CD009593.

14. García-Basteiro AL, DiNardo A, Saavedra B, et al. Point of care diagnostics for tuberculosis. Pulmonology 2018;24:73-85.

15. Pang Y, Dong H, Tan Y, et al. Rapid diagnosis of MDR and XDR tuberculosis with the MeltPro TB assay in China. Sci Rep 2016;6:25330.

16. Sharma SK, Kohli M, Yadav RN, et al. Evaluating the diagnostic accuracy of xpert MTB/RIF assay in pulmonary tuberculosis. PLoS One 2015;10:1-9.

17. Shrestha P, Arjyal A, Caws M, et al. The application of GeneXpert MTB/RIF for smear-negative TB diagnosis as a feepaying service at a south Asian general hospital. Tuberc Res Treat 2015;2015:1-6.

18. Dowdy DW, Cattamanchi A, Steingart KR, Pai M. Is scale-up worth it? Challenges in economic analysis of diagnostic tests for tuberculosis. PLoS Med 2011;8:e1001063.

19. Silva DR, Sotgiu G, D'Ambrosio L, et al. Diagnostic performances of the Xpert MTB/RIF in Brazil. Respir Med $2018 ; 134: 12-5$. 